\title{
Nano-graphene oxide composite for in vivo imaging
}

This article was published in the following Dove Press journal:

International Journal of Nanomedicine

Sung-Chan Jang, ${ }^{1,2, *}$ Sung-Min Kang, ${ }^{1, *}$ Jun Young Lee, ${ }^{3, *}$ Seo Yeong Oh,' AT Ezhil Vilian, ${ }^{4}$ Ilsong Lee, ${ }^{1,2}$ Young-Kyu Han, ${ }^{4}$ Jeong Hoon Park, ${ }^{3}$ Wan-Seob Cho, ${ }^{5, *}$ Changhyun Roh, ${ }^{2,6}$

\section{Yun Suk Huh'}

'Department of Biological Engineering, Biohybrid Systems Research Center (BSRC), Inha University, Incheon, ${ }^{2}$ Biotechnology Research Division, ${ }^{3}$ Radiation Instrumentation Research Division, Advanced Radiation Technology Institute (ARTI), Korea Atomic Energy Research Institute (KAERI), Jeongeup, ${ }^{4}$ Department of Energy and Materials Engineering, Dongguk University, Seoul, ${ }^{5}$ Laboratory of Toxicology, Department of Medicinal Biotechnology, College of Health Sciences, Dong-A University, Busan, ${ }^{6}$ Radiation Biotechnology and Applied Radioisotope Science, University of Science and Technology (UST), Daejeon, Republic of Korea

*These authors contributed equally to this work
Correspondence: Yun Suk Huh Department of Biological Engineering, Biohybrid Systems Research Center (BSRC), Inha University, 100 Inha-ro, Incheon 222I2, Republic of Korea

Tel +82 328609177

Email yunsuh.huh@inha.ac.kr

Changhyun Roh

Biotechnology Research Division, Advanced Radiation Technology Institute (ARTI), Korea Atomic Energy Research Institute (KAERI), 29 Geumgu-gil, Jeongeup, Jeonbuk 56212, Republic of Korea

Tel +8263570 3133

Email chroh@kaeri.re.kr
Introduction: Positron emission tomography (PET) tracers has the potential to revolutionize cancer imaging and diagnosis. PET tracers offer non-invasive quantitative imaging in biotechnology and biomedical applications, but it requires radioisotopes as radioactive imaging tracers or radiopharmaceuticals.

Method: This paper reports the synthesis of ${ }^{18} \mathrm{~F}-\mathrm{nGO}-\mathrm{PEG}$ by covalently functionalizing PEG with nano-graphene oxide, and its excellent stability in physiological solutions. Using a green synthesis route, $\mathrm{nGO}$ is then functionalized with a biocompatible PEG polymer to acquire high stability in PBS and DMEM.

Results and discussion: The radiochemical safety of ${ }^{18} \mathrm{~F}-\mathrm{nGO}-\mathrm{PEG}$ was measured by a reactive oxygen species and cell viability test. The biodistribution of ${ }^{18} \mathrm{~F}-\mathrm{nGO}-\mathrm{PEG}$ could be observed easily by PET, which suggested the significantly high sensitivity tumor uptake of ${ }^{18} \mathrm{~F}-\mathrm{nGO}-\mathrm{PEG}$ and in a tumor bearing CT-26 mouse compared to the control. ${ }^{18} \mathrm{~F}-\mathrm{nGO}-\mathrm{PEG}$ was applied successfully as an efficient radiotracer or drug agent in vivo using PET imaging. This article is expected to assist many researchers in the fabrication of ${ }^{18} \mathrm{~F}$-labeled graphene-based bio-conjugates with high reproducibility for applications in the biomedicine field.

Keywords: graphene oxide, nanocomposite, imaging, radiotracer

\section{Introduction}

Cancer is a leading cause of death and remains a great challenge to global health care. ${ }^{1}$ Mortality, however, has decreased, owing to a better understanding of cancer biology, improved diagnostic methods, and early detection of cancer through screening based on imaging. ${ }^{2}$ Cancer imaging forms an essential part of cancer clinical protocols and can provide morphological, structural, metabolic, and functional information. The imaging of specific tumor targets that are associated with cancer should allow earlier diagnosis and better management of oncology patients. ${ }^{3}$

Positron-emission tomography (PET) is a highly sensitive noninvasive technology that is ideally suited for preclinical and clinical imaging of cancer biology, in contrast to anatomical approaches. The technique is a noninvasive quantitative imaging technology that can monitor presymptomatic biochemical events early in the course of a disease $\mathrm{e}^{3-5}$ and improve disease detection, therapeutic monitoring, and treatment efficacy. ${ }^{6}$ The high sensitivity of the PET technique also makes it suitable for addressing questions fundamental to drug development for oncology, cardiology, neurosciences, and inflammatory diseases. ${ }^{7}$ PET is a powerful clinical imaging technique that is widely used for diagnostic applications in clinical oncology, owing to its unrivalled sensitivity and quantitative accuracy. ${ }^{3}$

PET radiotracers have been used to quantify biochemical processes in cancer patients and to fabricate bioactive molecules tagged with short-lived positron-emitting 
radionuclides, such as ${ }^{18} \mathrm{~F}$ (half-life $\left[t_{1 / 2}\right] 110$ minutes), ${ }^{11} \mathrm{C}$ ( $t_{1 / 2} 20$ minutes), and ${ }^{64} \mathrm{Cu}\left(t_{1 / 2} 12.7\right.$ hours). ${ }^{4,8,9}$ However, the limited $t_{1 / 2}$ of such radiotracers (typically only a few hours) necessitates dose-on-demand production at manufacturing sites in close proximity to the PET scanners. Therefore, the synthesis of PET radiotracers does not fit the typical drugmanufacturing paradigm. To address this, regulations specifically governing the current good manufacturing practice of PET radiotracers have been developed in recent years. ${ }^{10-12}$ To achieve the best contrast for PET imaging, several key issues need to be taken into consideration, such as how to choose the appropriate isotopes as positron emitters and nanoparticles as radiotracers, what chemical reactions can be utilized to improve the labeling efficiency, and how to functionalize the nanoparticles. ${ }^{13}$

Nanomaterials have unique properties, and have attracted considerable interest in biomedical applications. Nanomaterials with diameters of 10-100 $\mathrm{nm}$ can be extravasated through the endothelial cell layers and interact with cell structures of various tissues, due to the enhanced permeability and retention (EPR) effect. ${ }^{13}$ General features of tumors include leaky blood vessels and poor lymphatic drainage. While imaging tracers may diffuse non-specifically, a radiotracer can extravagate into tumor tissue via the leaky vessels by the EPR effect. ${ }^{14,15}$ The size of the nanomaterials suggests that the threshold vesicle size for extravasation into tumors is $\sim 400 \mathrm{~nm} .{ }^{16}$ Many researchers have shown that particles with diameters $<200 \mathrm{~nm}$ are more effective. ${ }^{16-18}$ In addition, the large surface area:volume ratio renders nanoparticles with the ability to be readily loaded with a variety of diagnostic and/or therapeutic agents as theranostics for disease detection and treatment. ${ }^{14}$

Traditional chelation methods have been used to incorporate various radionuclides on a radiotracer, but these methods are compromised by the potential for the loss of the radionuclide, due to cleavage of the linker between the particle and the chelator, decomplexation of the metal, and possible altered pharmacokinetics of the nanomaterials. This methodology, however, creates some concerns for in vivo diagnostic imaging. First, the radiometal chelator attached to nanomaterials might alter the physicochemical properties of the nanomaterials. Second, the radiometal chelator moiety may dissociate from the surface of the nanomaterials through either dissociation of the ligand from the metal core or transchelation of the metal into biological fluids, which could cause a mismatch in location between radionuclides and nanomaterials. Third, conjugation is challenging when the original surface is not conjugation-friendly. These strategies suffer from either a lack of operational simplicity or broad applicability, which has hampered translational studies and the rapid progress of PET imaging.

Functionalized graphene oxide (GO; 2-D)-based nanocomposites have attracted attention in biomedical applications in recent years, because of their unique and highly enriched physical and chemical properties, ${ }^{19-25}$ such as excellent biocompatibility, ready cellular uptake, flexible chemical modifications, unique optical properties, and thermal and electrical conductivity. In recent years, graphenebased nanomaterials have also attracted significant interest in biological fields, such as biomedicine, biosensors, drug delivery and bioimaging.

This paper describes the straightforward synthesis of intrinsically radioactive fluorinated labeled nano-GO $\left({ }^{18} \mathrm{~F}\right.$ nGO-polyethylene glycol [PEG]) by directly incorporating ${ }^{18} \mathrm{~F}$ into an nGO-PEG nanostructure with nGO-PEG as the precursor. ${ }^{18} \mathrm{~F}$-nGO-PEG was simply synthesized by mixing GO with ${ }^{18} \mathrm{~F}$ ions to form intrinsically radiolabeled $\mathrm{GO}$ without the assistance of any chelators. Due to its versatile surface functionalization and high surface area, $\mathrm{nGO}$ can be easily functionalized by small molecules, nanoparticles, or drugs to obtain graphene-based nanomaterials for bioimaging applications. Therefore, it is important to develop reliable, chelator-free, intrinsically radioactive ${ }^{18} \mathrm{~F}-\mathrm{nGO}-\mathrm{PEG}$ for in vivo imaging. The functionalized nanomaterial can also be used for potential drug-delivery applications. ${ }^{18} \mathrm{~F}-\mathrm{nGO}-\mathrm{PEG}$ has solved the problems of aggregation of particles and loss of radioactivity previously encountered with graphene-based nanomaterials kept in physiological buffer or inserted into animals. This study demonstrates that PEGylated nGO can act as an excellent biocompatible radiotracer for loading imaging molecules, and may become a great platform to realize the imaging of tumor cells. ${ }^{18} \mathrm{~F}-\mathrm{nGO}-\mathrm{PEG}$ was injected subcutaneously into living animals, and excellent detection sensitivity and multicolor capability were achieved. These results offer an innovative development in ultrasensitive detection of ${ }^{18} \mathrm{~F}$-nGO-PEG tumor uptake and the tumor imaging of biomarkers involved in cancer.

\section{Materials and methods}

Nanosized graphite powder (Nano G-400) was obtained from Graphene Supermarket (Calverton, NY, USA). PEG (six-armed PEG-NH, $10 \mathrm{kDa}$ ) was obtained from SunBio (Gunpo, Republic of Korea). Potassium persulfate, phosphorous pentoxide, potassium permanganate, ferrous sulfate, potassium ferrocyanide, sulfuric acid, hydrochloric acid, hydrofluoric acid, trifluoroacetic acid (TFA), sodium 
hydroxide, $2^{\prime} 7^{\prime}$-dichlorofluorescein diacetate (DCF-DA), and ethyl(dimethylaminopropyl) carbodiimide (EDC) were purchased from Sigma-Aldrich (St Louis, MO, USA). 1-Chloro2,2,2-trifluoroethyl difluoromethyl ether (isoflurane) was supplied by Terrell.

\section{Characterization of nGO, nGO-PEG, and F-nGO-PEG}

Field-emission transmission electron microscopy (TEM; JEM-2100F; JEOL, Tokyo, Japan) was performed at an accelerating voltage of $300 \mathrm{kV}$. Ultraviolet (UV)-visible spectra were recorded on a Jasco V-770. Fourier-transform infrared (FT-IR) spectra were recorded using a Jasco FT/IR-6600. X-ray diffraction (XRD; D/Max-2500; Rigaku, Tokyo, Japan) patterns were collected using $\mathrm{CuK} \alpha$ radiation. X-ray photoelectron spectroscopy (K-Alpha; Thermo Fisher Scientific, Waltham, MA, USA) was performed using an Al X-ray source. Thin-layer chromatography (TLC) was performed on silica-gel $60 \mathrm{~F}_{254}$ glass plates purchased from Merck Millipore (Billerica, MA, USA). PET images were taken using micro-PET (Genesys4; Sofie Biosciences, Culver City, CA, USA).

\section{Synthesis and functionalization of $n G O$, nGO-PEG, and F-nGO-PEG}

Chemical exfoliation of nGO was achieved using the modified Hummers method. Chemical exfoliated nGO (10 mg) was dispersed in $1 \mathrm{~mL}$ water. Subsequently, $1.8 \mathrm{~g} \mathrm{NaOH}$ was added to the $\mathrm{nGO}$ solution and the mixture heated at $55^{\circ} \mathrm{C}$ for 4 hours. A $37 \%$ (v:v) $\mathrm{HCl}$ solution ( $4 \mathrm{~mL}$ ) was then added to the solution to neutralize the $\mathrm{NaOH}$. The product was washed four times with deionized (DI) water to remove the salt. The as-obtained product was washed using centrifugation at 13,000 rpm for 10 minutes. PEG-NH $(50 \mathrm{mg})$ was added to the base-treated $\mathrm{nGO}$ solution $(10 \mathrm{mg} / \mathrm{mL}$ and $5 \mathrm{~mL})$ in a $25 \mathrm{~mL}$ vial. The suspension was sonicated vigorously in a water bath at room temperature for 15 minutes, and $10 \mathrm{mg}$ EDC was added as a catalyst. The solution was sonicated for 30 minutes at room temperature, and another $10 \mathrm{mg}$ EDC was then added. Subsequently, the mixture solution was stirred overnight at room temperature. The final mixture solution was washed five or six times by repeating the centrifuging and water-addition steps, to completely remove the excess PEG and EDC in the obtained nGO-PEG solution. The $10 \mathrm{mg} / \mathrm{mL}$ nGO-PEG suspension ( $1 \mathrm{~mL}$ ) was treated in a glass vial on a hotplate at $80^{\circ} \mathrm{C}$ for 1 hour. A $100 \mu \mathrm{L}$ solution of hydrofluoric acid was also added to make acidic conditions. The resulting mixture was left to stand at $80^{\circ} \mathrm{C}$ for 1 hour. The mixture was rinsed with DI water. F-nGO-PEG was obtained for further analysis.

\section{Toxicity assays ROS test}

To make the reactive oxygen species (ROS) test solution, in the DCFH assay $100 \mu \mathrm{L}$ of DCFH-DA was hydrolyzed chemically to DCFH with $4 \mathrm{~mL} 0.01 \mathrm{~N} \mathrm{NaOH}$ for 30 minutes in a darkroom. After adding $20 \mathrm{~mL}$ PBS for neutralization, $100 \mu \mathrm{L}$ nGO and nGO-PEG each were placed separately into $100 \mu \mathrm{L}$ HRP (2.9 unit/mL) and $100 \mu \mathrm{L}$ of neutralized DCF-DA. The mixtures were then incubated for 15 minutes in a darkroom. The samples were then centrifuged at 13,000 rpm for 15 minutes. The supernatant fraction was dispensed in $200 \mu \mathrm{L}$ per well of 96 wells. Absorbance was measured at $485 \mathrm{~nm}$ using a microplate reader. ${ }^{26} \mathrm{~A}$ blank (no nanomaterial added) and a positive control $\left(\mathrm{H}_{2} \mathrm{O}_{2}\right)$ were used in the assay, both in triplicate. The fluorescence values obtained for the nanomaterial were converted to $\mathrm{H}_{2} \mathrm{O}_{2}$ equivalents against a calibration curve of $\mathrm{H}_{2} \mathrm{O}_{2}$ in the range of $0-9 \mu \mathrm{M}$ of $\mathrm{H}_{2} \mathrm{O}_{2}$, with $r^{2}=0.98$. The conversion of arbitrary fluorescence units to a biologically meaningful ROS value is desirable, and one approach used for the nanomaterial was converted to $\mathrm{H}_{2} \mathrm{O}_{2}$ equivalents. Because $\mathrm{H}_{2} \mathrm{O}_{2}$ cannot oxidize DCFH independently, the enzyme HRP was added to catalyze the generation of $\mathrm{OH}$ radicals. Therefore, $\mathrm{HRP}$ was added only when $\mathrm{H}_{2} \mathrm{O}_{2}$ was used as a positive control and for the standards.

\section{Cell lines}

Human breast cancer MCF7 cells were cultured in RPMI 1640 medium supplemented with 10\% FBS and L-glutamine. Mouse colon cancer CT26 cells were cultured in DMEM with 10\% FBS, 4 mM L-glutamine, 4,500 mg/L glucose, and sodium pyruvate. All cancer cell lines were obtained from the Korean Cell Line Bank (Seoul, Republic of Korea) and maintained at $37^{\circ} \mathrm{C}$ in a humidified atmosphere containing $5 \% \mathrm{CO}_{2}$.

\section{In vitro cytotoxicity assay}

The MCF7 cell suspension ( $2 \mathrm{~mL}$ ) was dispensed in a 24-well plate $\left(3.98 \times 10^{4}\right.$ cells/well). Subsequently, $200 \mu \mathrm{L}$ of different concentrations $(0,1,10,20,50 \mu \mathrm{g} / \mathrm{mL})$ of nGO and nGOPEG was added to $1,800 \mu \mathrm{L}$ of the cell-culture media and incubated for 24 hours in a humidified incubator (at $37^{\circ} \mathrm{C}$, $5 \% \mathrm{CO}_{2}$ ). Following the $\mathrm{nGO}$ and nGO-PEG treatments, the cells were washed with PBS and treated with $10 \mu \mathrm{L}$ of a CCK- 8 solution and $2 \mathrm{~mL}$ of cell-culture media in each well of the plate. Cells were incubated for 3 hours in an incubator 
(at $37^{\circ} \mathrm{C}, 5 \% \mathrm{CO}_{2}$ ). The supernatant fraction was dispensed at $200 \mu \mathrm{L}$ per well in a 96-well plate. Absorbance was measured at $450 \mathrm{~nm}$ using a microplate reader.

\section{Cell-uptake test}

Levels of ${ }^{18} \mathrm{~F}$-nGO-PEG expression in two different human cancer cell lines (MCF7) and a mouse cancer cell line (CT26) were determined. Briefly, ${ }^{18} \mathrm{~F}-\mathrm{nGO}-\mathrm{PEG}$ uptake was measured by incubating the cells with approximately $5 \mu \mathrm{Ci}{ }^{18} \mathrm{~F}$-nGO-PEG in $2 \mathrm{~mL}$ serum-free medium at $37^{\circ} \mathrm{C}$ for 15-120 minutes. After incubation, cells were quickly washed twice with $2 \mathrm{~mL}$ cold PBS. Cells were detached with $200 \mu \mathrm{L}$ of $1 \%$ sodium dodecyl sulfate and radioactivity measured using a $\gamma$-counter. ${ }^{18} \mathrm{~F}$-nGO-PEG uptake was expressed as percentage injected dose. ${ }^{27}$

\section{Radiolabeling of nGO-PEG with ${ }^{18} \mathrm{~F}$}

nGO-PEG was labeled with the ${ }^{18} \mathrm{~F}$ radionuclide (obtained RFT-30 MeV Cyclotron facility at the Korea Atomic Energy Research Institute, Jeongeup, Republic of Korea using a standard chloramine-T oxidation method. In recent years, the ${ }^{18} \mathrm{O}(\mathrm{p}, \mathrm{n}){ }^{18} \mathrm{~F}$ reaction on highly enriched ${ }^{18} \mathrm{O}$ targets (both gaseous and $\mathrm{H}_{2}{ }^{18} \mathrm{O}$ ) has been used extensively. ${ }^{28}$ The ${ }^{18} \mathrm{~F}$ radioisotope $\left(t_{1 / 2} 109.7\right.$ minutes; $\mathrm{I}_{\beta+} 97 \%$; $\left.\mathrm{E}_{\beta+} 0.63 \mathrm{MeV}\right)$ is produced in a cyclotron via the ${ }^{18} \mathrm{O}(\mathrm{p}, \mathrm{n}){ }^{18} \mathrm{~F}$ nuclear reaction by bombarding ${ }^{18} \mathrm{O}$ water with a high-energy proton beam, which is then delivered to the radiochemistry laboratory in a solution of ${ }^{18} \mathrm{O}$ water to conduct the radiochemical reactions. Briefly, $100 \mu \mathrm{Ci}{ }^{18} \mathrm{~F}$ and $0.1 \mathrm{~mL}$ trifluoroacetic acid were added to a solution of nGO-PEG ( $1 \mathrm{mg} / \mathrm{mL}, 1 \mathrm{~mL})$. The mixture was then reacted in a $\mathrm{pH} 5.5$ trifluoroacetic acid solution (10\%) for 1 hour at $80^{\circ} \mathrm{C}$ to make an acidic condition. Excess ${ }^{18} \mathrm{~F}$ was removed by centrifugation filtration through the filters $(0.2 \mu \mathrm{m})$ washing several times with a saline solution until there was no detectable radioactivity in the filtration solution. For the radiolabeling stability assay, $1 \mathrm{~mL}$ obtained ${ }^{18} \mathrm{~F}-\mathrm{nGO}-$ PEG ( $1 \mathrm{mg} / \mathrm{mL}$ ) was taken. The leftover ${ }^{18} \mathrm{~F}-\mathrm{nGO}-\mathrm{PEG}$ after washing was collected for $\gamma$-counting to measure the amount of ${ }^{18} \mathrm{~F}$ retained on the $\mathrm{nGO}-\mathrm{PEG}$ nanoparticles.

\section{Preparation for in vivo experiment and PET imaging}

Immediately before transfection, the serum-containing medium was removed and replaced with PBS. The CT26 tumors were grown in the right thighs of nude mice (20-25 g) by injecting $10^{7}$ tumor cells suspended in $100 \mu \mathrm{L}$ PBS subcutaneously. When the tumors had grown to $10-15 \mathrm{~mm}$ in diameter (Figure S1), the tumor-bearing mice were injected intravenously with $100 \mu \mathrm{L}{ }^{18} \mathrm{~F}$-nGO-PEG or ${ }^{18} \mathrm{~F}$ solution $>100 \mu \mathrm{Ci}$ for PET scanning, and X-ray imaging was performed at different time points postinjection. All animal studies were conducted in compliance with animal experimental guidelines, and ethics approval was obtained from the Korea Atomic Energy Research Institute (IACUC-2015-004).

\section{Results and discussion Radiolabeling of $n$ GO-PEG with ${ }^{18} \mathrm{~F}$}

Figure 1 shows a homogeneous dispersion of nGO-PEG in a solution synthesized directly from $\mathrm{nGO}$ using a polymeric surfactant (PEG). In this study, nGO was functionalized with PEG to improve the dispersion and their physiological stability. ${ }^{29,30}$ Carboxyl functional groups on nGO were conjugated covalently with amine-terminated PEG $(10 \mathrm{kDa})$ and $N$-(3-dimethylaminopropyl)- $N$-ethylcarbodiimide (EDC) hydrochloride. This is the most commonly used approach in our group to prepare small PEGylated $\mathrm{nGO}(50-100 \mathrm{~nm})$ (Figure 1A and B). ${ }^{30}$ Compared to GO, nGO-PEG was much smaller, owing to ultrasonication involved during the PEG-conjugation step, which greatly breaks down the nanosheets. The GO surface contains oxygen functional groups (carboxyl and hydroxyl groups) bound to the radiohalide via a chemical reaction without a chelator, which was to be used as an excellent radiotracer (Figure S2). The resulting nGO-PEG exhibited excellent stability in various physiological solutions, including DI water, PBS, and cell medium. In this labeling method, ${ }^{18} \mathrm{~F}$ atoms were anchored to the defect sites or edges of the nGO-PEG sheet (Figure 1C). ${ }^{18} \mathrm{~F}$ was introduced to nGO-PEG, which can oxidize fluorine ions to $\mathrm{F}$ atoms to attack oxygen groups on the graphene surface via a chemical reaction. ${ }^{18} \mathrm{~F}$ has been applied widely in the PET imaging of in vivo tumor cells, because it is a $\gamma$-ray emitter. The ${ }^{18} \mathrm{~F}$-labeled nGO-PEG exhibited a high radiolabeling yield, as well as great radiolabeling stability in an aqueous solution at room temperature. As such, the radionuclide ${ }^{18} \mathrm{~F}$ was introduced to label $\mathrm{nGO}-\mathrm{PEG}$ via chemical reaction with both high efficiency and stability.

\section{Studying the reaction of $n G O$ with PEG}

TEM was used to reveal the morphological characteristics of nGO and nGO-PEG. From the TEM image (Figure 2), nGO exhibited the wrinkled paper-like structure of graphene sheets with diameters of $\sim 50-100 \mathrm{~nm}$ (Figure 2A and B). On the other hand, nGO-PEG revealed uniform disk morphology 
A nGo
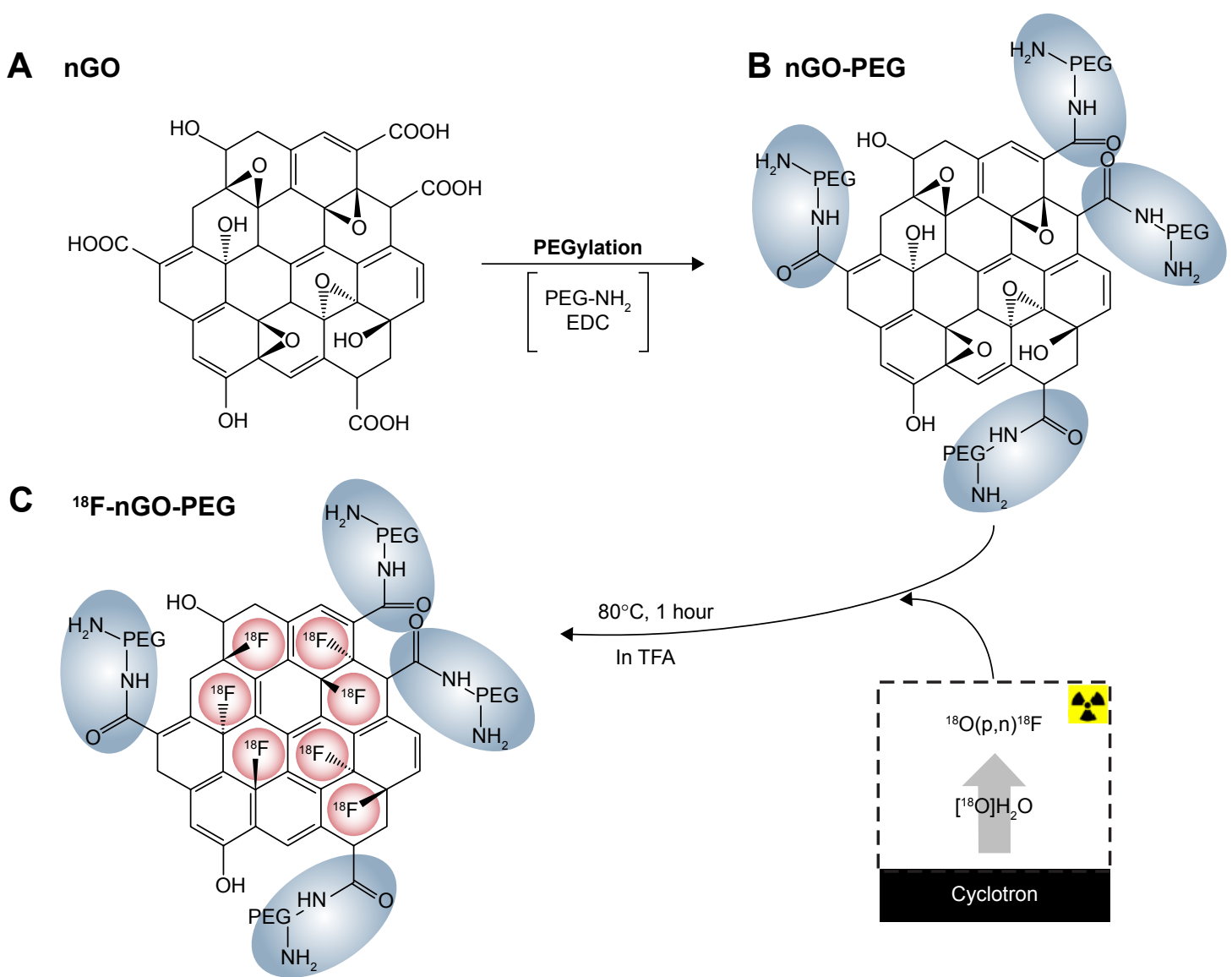

Figure I Illustration of ${ }^{18} \mathrm{~F}-\mathrm{nGO}-\mathrm{PEG}$.

Notes: (A) Chemical structure of nGO. nGO has an oxygen group at the basal plane and edge of graphene. (B) PEGylation of nGO, as well as nGO-PEG, which is conjugated six-armed PEG. (C) Chemical structure of ${ }^{18} \mathrm{~F}$-nGO-PEG induced by the cyclotron equipment.

Abbreviations: nGO, nano-graphene oxide; PEG, polyethylene glycol; EDC, ethyl(dimethylaminopropyl) carbodiimide; TFA, trifluoroacetic acid.

with a mean diameter of $\sim 100 \mathrm{~nm}$ (Figure $2 \mathrm{C}$ and $\mathrm{D}$ ). The as-obtained nGO-PEG showed amine-terminated PEG adsorbed on the surface of nGO.

nGO-PEG fabricated from nGO was used to measure surface-charge changes using $\zeta$-potential. Initially, the nGO $\zeta$-potential change was $-70 \mathrm{mV}$. This suggests that $\mathrm{nGO}$ has a negative charge on the surface, because nGO has a number of oxygen functional groups on the surface. After PEGylation on the nGO surface, the charge was $-48 \mathrm{mV}$, which shows that the amine-terminated nGO had shifted to the positively charged direction on the final product surface (Figure 2E). The more positively charged nGO-PEG might be favorable as an anionic growth factor in bioimaging and drug carriers for delivery applications. The phase and crystal structure of nGO-PEG were characterized by XRD. The XRD pattern of nGO prepared revealed a broad reflection at $11.521^{\circ}$, which can be assigned to the (002) reflection of nGO (Figure 2F). The broad XRD peaks of PEG on the $\mathrm{nGO}$ sheet suggested that the (002)-diffraction peak shifted to a lower angle with
PEG on the surface of the nGO sheets. These results suggest that the interlayer spacing becomes larger with the addition of PEG decorated on the $\mathrm{nGO}$ sheets. These results strongly suggest successful conjugation of PEG onto nGO.

\section{Stability studies in physiological media and viability assay}

The radiochemical stability of nGO and nGO-PEG was studied in various media, including PBS (pH 7.4) and cellculture media. nGO-PEG was soluble in DI water, while nGO was not dispersed in PBS or DMEM..$^{31}$ Therefore, nGO aggregated and precipitated in the presence of salts in the bottom of the DI water, PBS, and DMEM (Figure 3A). On the other hand, the biocompatibility of the amine-functionalized nGO-PEG was utilized. Hydrophilic PEG chains can improve nanomaterial stability in many biological solutions. This might be because amine-terminated PEG shows covalent binding to carboxylic acid groups on $\mathrm{nGO}$, due to PEG being soluble in DI water, PBS, and DMEM. Among the different 

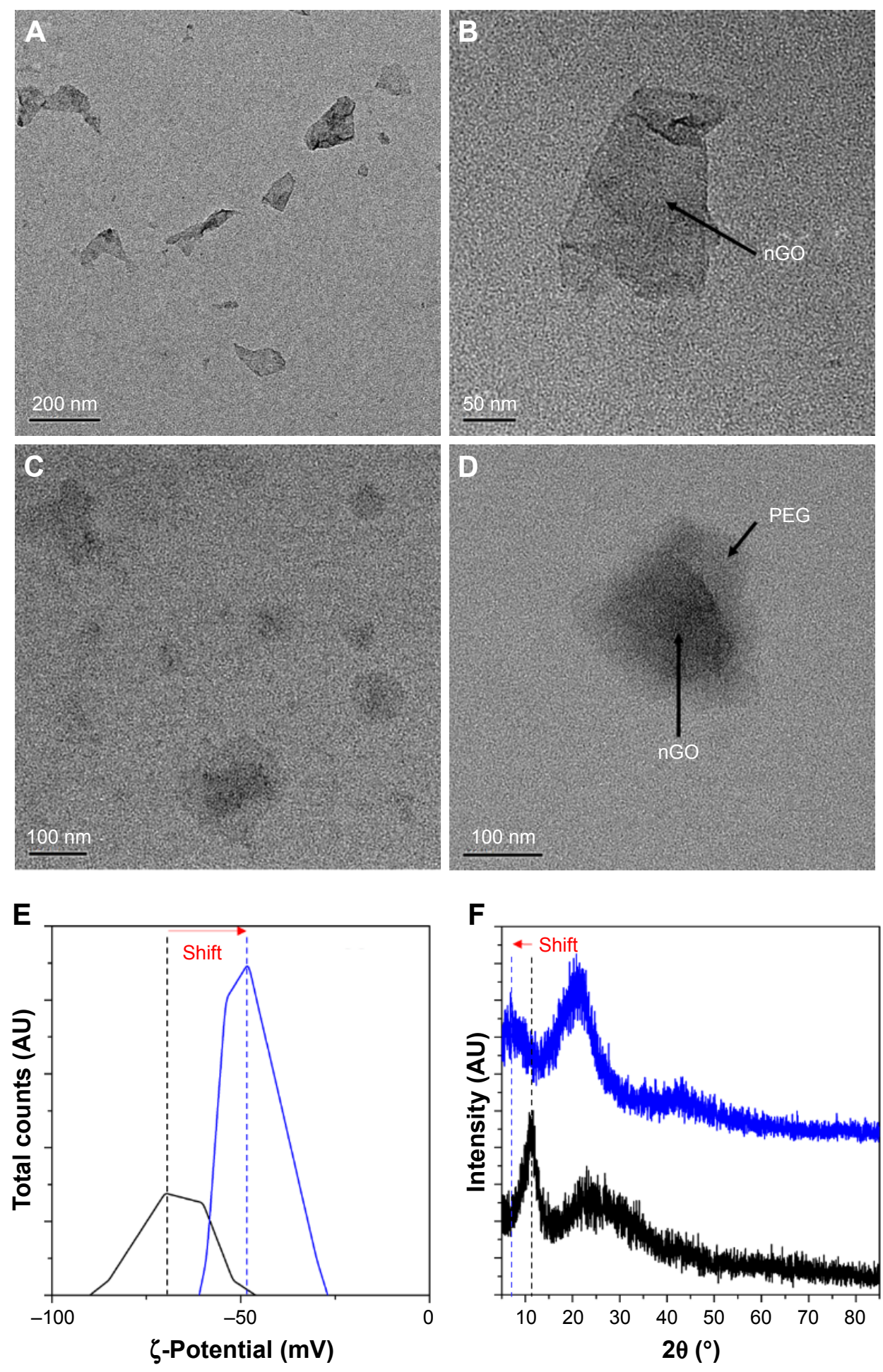

nGO - nGO-PEG

Figure 2 TEM images of (A, B) nGO and (C, D) nGO-PEG; (E) $\zeta$-potential peak; (F) XRD patterns of nGO and nGO-PEG.

Abbreviations: $\mathrm{nGO}$, nano-graphene oxide; PEG, polyethylene glycol; TEM, transmission electron microscopy; XRD, X-ray diffraction.

solvents tested, nGO in the presence of PEG was the best nanomaterial for use in PET imaging (Figure 3B). UV-visible spectra of nGO and nGO-PEG in DMEM were measured to confirm stability. The black lines indicated the initial state, and red lines were shown after centrifuge. This stability clearly suggests that nGO-PEG can be dispersed in biological environments (Figure 3C). This study is expected to help scientists in this field assemble nGO-PEG nanomaterials with 

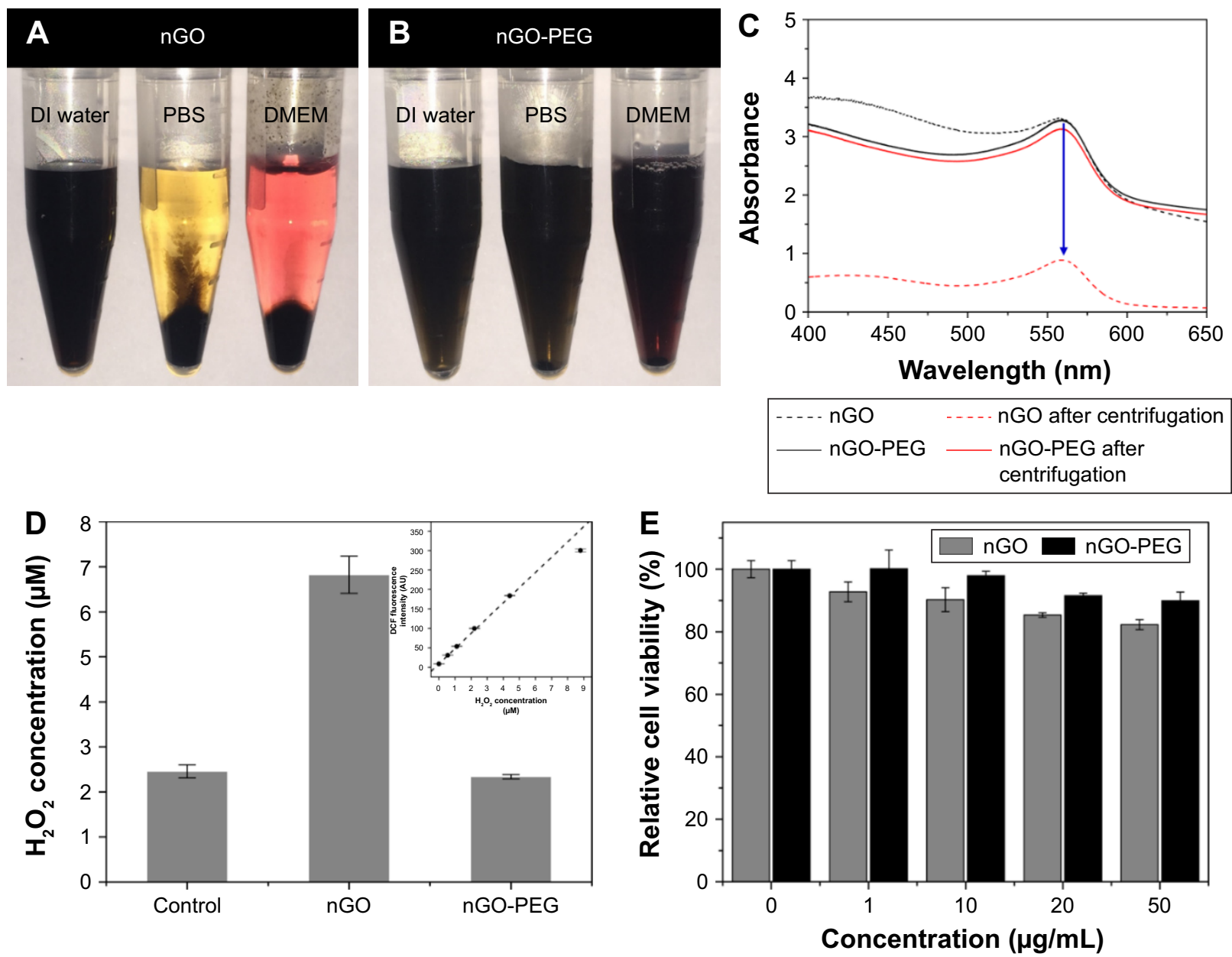

Figure 3 Characterization of $n G O$ and $n G O-P E G$.

Notes: (A, B) The photographs show nGO and nGO-PEG in different solutions: DI water, PBS buffer, and DMEM solution. Photos taken after I0,000 rpm for 5 minutes of $n G O(\mathbf{A})$ and nGO-PEG (B). (C) Ultraviolet-visible spectra of nGO and nGO-PEG in DMEM. Black lines indicate initial state, red lines after centrifugation. (D) ROS test of nGO and nGO-PEG compared with PBS as a control sample. The inset show standard curve of $\mathrm{H}_{2} \mathrm{O}_{2}$ concentration vs DCF fluorescence intensity to measure $\mathrm{H}_{2} \mathrm{O}_{2}$ concentration. (E) Cell-viability study. MCF7 cancer cells after incubation with nGO or nGO-PEG.

Abbreviations: DCF, dichlorofluorescein; DI, deionized; nGO, nano-graphene oxide; PEG, polyethylene glycol; ROS, reactive oxygen species.

high reproducibility for numerous applications in biomedicine and biotechnology.

\section{Toxicity of nGO-PEG in vitro}

This study investigated the ability of nGO-PEG to induce the formation of intracellular ROS by monitoring the increasing fluorescence of DCF-DA. Figure 3D shows ROS levels after exposure to $\mathrm{nGO}$ and $\mathrm{nGO}-\mathrm{PEG}$ for 2 hours relative to the untreated control. nGO showed a 2.6-fold increase in ROS relative to the controls. nGO-PEG did not induce detectable ROS formation compared to the untreated control.

The cytotoxicity of the nGO and nGO-PEG on breast cancer cells (MCF7) was examined by MTT (CCK-8). The MCF7 cells were incubated with $\mathrm{nGO}$ and $\mathrm{nGO}-\mathrm{PEG}$ in a $\mathrm{CO}_{2}$ incubator for 24 hours. nGO-PEG significantly suppressed the proliferation of breast cancer cells, while no cytotoxicity was detected at high concentrations of $\mathrm{nGO}$ and $\mathrm{nGO}-\mathrm{PEG}$
(Figure 3E). Interestingly, no obvious cytotoxicity appeared after 24 hours of exposure of the animal cells to a high concentration $(50 \mu \mathrm{g} / \mathrm{mL})$ of nGO-PEG in CCK-8. Interestingly, these results showed that nGO has higher cytotoxicity than nGO-PEG. Finally, nGO-PEG was used as a drug carrier to test cellular biocompatibility.

\section{Measurements of $F$ concentration on nGO-PEG}

The chemical structure of the interactions between $\mathrm{F}$ and nGO-PEG surfaces was monitored by FT-IR spectroscopy (Figure 4A). The FT-IR spectrum of nGO showed peaks for hydroxy stretching $\left(\mathrm{O}-\mathrm{H}, 3,429 \mathrm{~cm}^{-1}\right), \mathrm{C}=\mathrm{O}$ stretching (carbonyl or carboxyl, $1,705 \mathrm{~cm}^{-1}$ ), aromatic $\mathrm{C}=\mathrm{C}$ stretching $\left(1,624 \mathrm{~cm}^{-1}\right)$, carboxy $\mathrm{C}-\mathrm{O}$ stretching $\left(1,397 \mathrm{~cm}^{-1}\right)$, epoxy $\mathrm{C}-\mathrm{O}$ stretching $\left(1,192 \mathrm{~cm}^{-1}\right)$, and alkoxy $\mathrm{C}-\mathrm{O}$ stretching $\left(1,058 \mathrm{~cm}^{-1}\right)$ as shown in black. In the red curve, the 
A

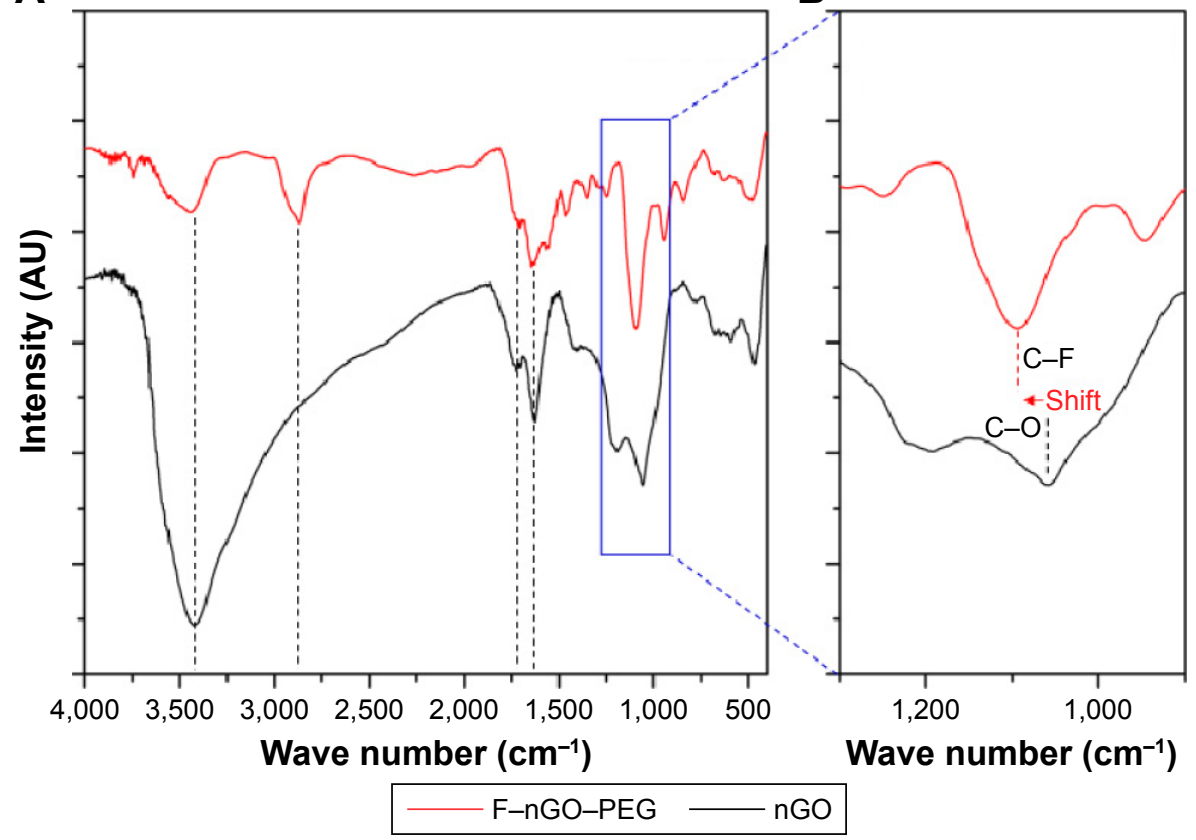

Figure 4 Characterization of nGO and F-nGO-PEG. (A) FT-IR; (B) magnified FT-IR spectra.

Abbreviations: FT-IR, Fourier-transform infrared; nGO, nano-graphene oxide; PEG, polyethylene glycol.

much-smaller peak at $3,429 \mathrm{~cm}^{-1}$ was because of the absence of $\mathrm{O}-\mathrm{H}$ bonds after $\mathrm{F}$ substitution; the new peak at $2,850 \mathrm{~cm}^{-1}$ may have been due to amine bonds related to $\mathrm{PEG}-\mathrm{NH}_{2}$. The characteristic absorption peak at $1,093 \mathrm{~cm}^{-1}$ was assigned to the vibrations of the $\mathrm{C}-\mathrm{F}$ band of the $\mathrm{F}$ functional group bound to the edge of the nGO-PEG surface (Figure 4B). Finally, this study confirmed that before-and-after $\mathrm{F}$ functionalization of the nGO-PEG successfully made up the effective attached $\mathrm{F}$ on the surface of $\mathrm{nGO}-\mathrm{PEG}$ radiotracers.

The chemical composition of fluorinated $\mathrm{nGO}$ and nGO-PEG materials was examined by X-ray photoelectron spectroscopy (Figure 5). The full-scan survey spectrum of F-nGO-PEG contained fluorine $\left(\mathrm{F}_{1 \mathrm{~s}}\right)$, carbon $\left(\mathrm{C}_{1 \mathrm{~s}}\right)$, and oxygen $\left(\mathrm{O}_{1 \mathrm{~s}}\right)$. The $\mathrm{F}_{1 \mathrm{~s}}$ peak intensity diminished gradually in nGO, while the $\mathrm{C}_{1 \mathrm{~s}}$ peak intensity increased considerably. These results show chemical exfoliation of graphite to nGO, and that the amount of fluorine diminished. The F-nGOPEG radiotracers showed an $\mathrm{F}_{1 \mathrm{~s}}$ peak at approximately $725 \mathrm{eV}$, indicating the chemical content of fluorine on the radiotracer surface.

\section{Radio-TLC to measure radioactivity of radiolabeled $n$ GO-PEG}

The labeling reaction was monitored by radio-TLC (EtOAc: $n$-hexane 1:1). The radiolabeling yield of ${ }^{18} \mathrm{~F}$-nGO-PEG was then determined at different time intervals using radio-TLC, where ${ }^{18} \mathrm{~F}-\mathrm{nGO}-\mathrm{PEG}\left(\mathrm{R}_{\mathrm{F}}=0 \mathrm{~mm}\right)$ could be distinguished easily from free ${ }^{18} \mathrm{~F}\left(\mathrm{R}_{\mathrm{F}} \approx 90 \mathrm{~mm}\right)$. The radiolabeling yield of ${ }^{18}$ F-nGO-PEG was $>96.06 \%$ after 1 hour's incubation, confirming the successful labeling of ${ }^{18} \mathrm{~F}$ to $\mathrm{nGO}-\mathrm{PEG}$ (Figure 6A).

\section{Cell-uptake studies}

To investigate in vitro cell-uptake behavior of ${ }^{18} \mathrm{~F}-\mathrm{nGO}$ PEG, MCF7 and CT26 cancer cells were incubated in the presence of nGO-PEG. After cells had been incubated with ${ }^{18} \mathrm{~F}$-nGO-PEG for 120 minutes, remarkably increased cell uptake was observed (Figure 6B). For cancer cells, such as MCF7 and CT26, similar uptake behavior was observed. This demonstrated that the uptake seemed to be cancer-cell type-independent.

\section{PET-imaging studies}

Here, ${ }^{18} \mathrm{~F}-\mathrm{nGO}-\mathrm{PEG}(100 \mu \mathrm{Ci}$ per mouse $)$ was administered intravenously into the mice and bioimaging performed using the micro-PET system (Genesys4). The free ${ }^{18} \mathrm{~F}$-treated mice and ${ }^{18} \mathrm{~F}$-nGO-treated mice were used as the control (Figures S3 and S4). It seemed that free ${ }^{18} \mathrm{~F}$ and ${ }^{18} \mathrm{~F}-\mathrm{nGO}$ show PET signal in vivo biodistribution profile, with high uptake in the bladder, blood, lungs, and main vessels. No signal was observed in tumor cells under in vivo conditions. ${ }^{18} \mathrm{~F}$-nGO-PEG was then injected intravenously into the female 

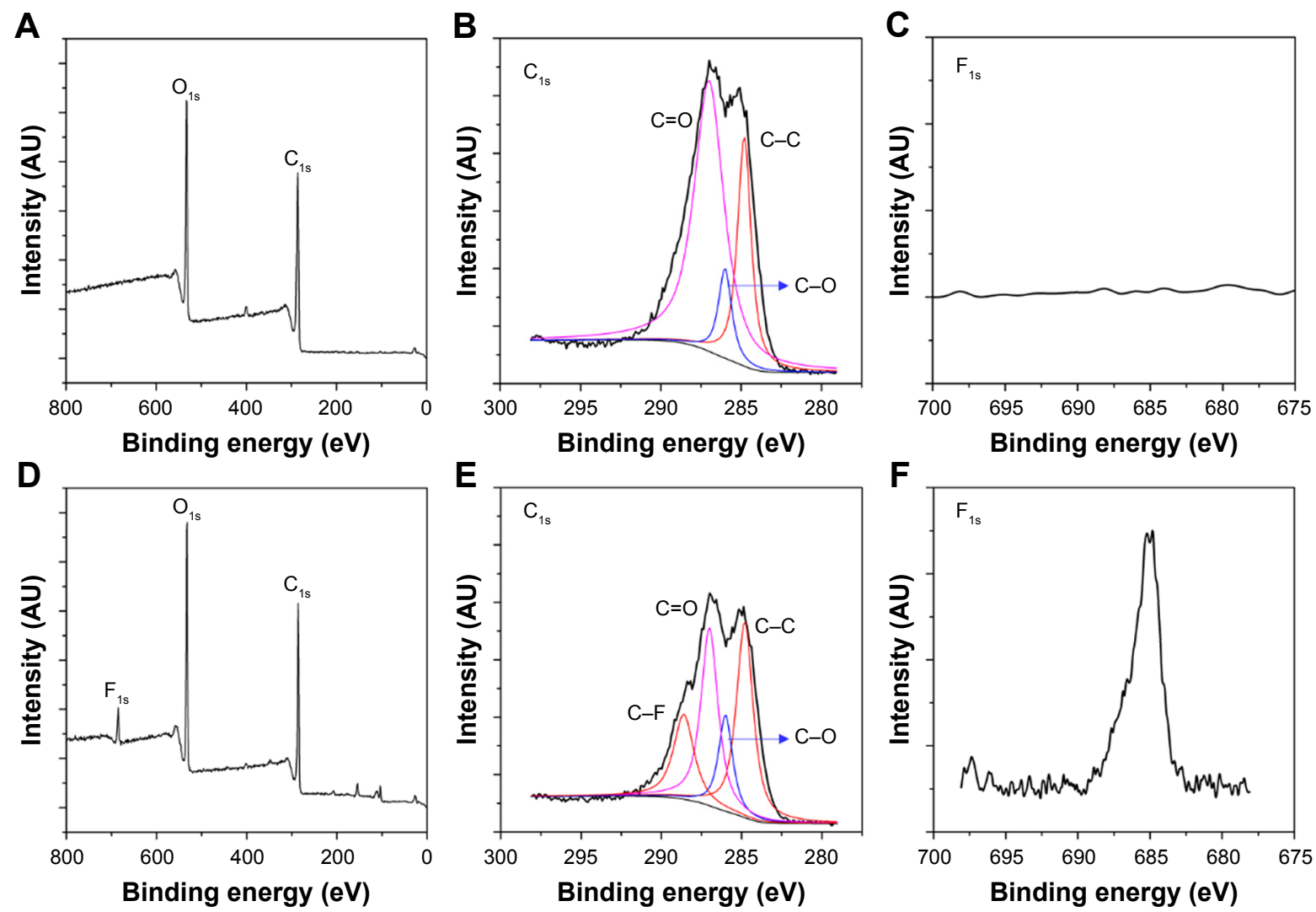

Figure 5 Survey $X$-ray photoelectron spectra.

Notes: (A) As-prepared nGO. (B) $C_{1 s}$ and (C) $F_{1 s}$ spectra of nGO. (D) As-prepared F-nGO-PEG. (E) $C_{1 s}$ and (F) $F_{1 s}$ spectrum of F-nGO-PEG.

Abbreviations: $n G O$, nano-graphene oxide; PEG, polyethylene glycol.

mice, and the image was monitored at various time points (15, 30, and 60 minutes) (Figure S5). After 15 minutes, an intact ${ }^{18} \mathrm{~F}-\mathrm{nGO}-\mathrm{PEG}$ tumor and bioimaging of the tumor cell were monitored (Figure 7). ${ }^{18} \mathrm{~F}-\mathrm{nGO}-\mathrm{PEG}$ accumulated passively in the tumor via the enhanced cell-EPR effect (Figure 7A). ${ }^{18} \mathrm{~F}-\mathrm{nGO}-\mathrm{PEG}$ was taken up rapidly into the early endosomes and lysosomes of CT26 tumor cells. Subsequently, ${ }^{18} \mathrm{~F}-$ nGO-PEG was injected into the tail veins of the mice and rapidly accumulated in endolysosomal compartments in vivo. Micro-PET imaging studies (Figure 7B) showed tumorimaging signals in an in vivo biodistribution profile, with high uptake in tumor cells in the right thighs of the nude mice.

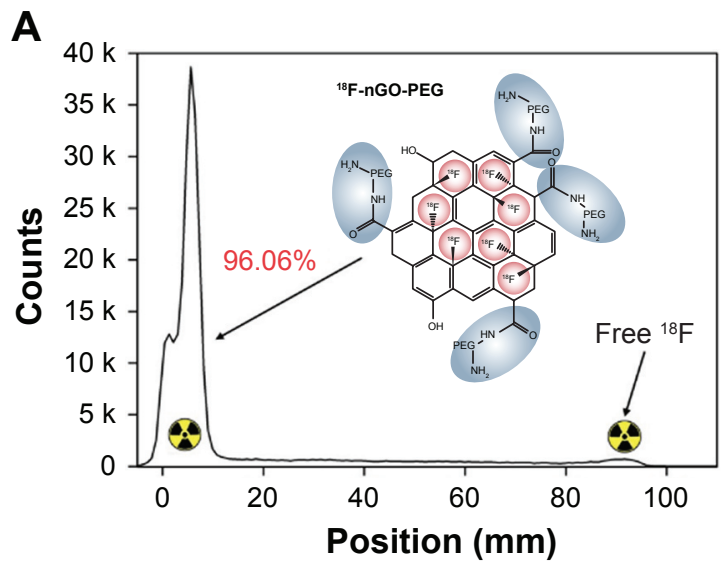

B

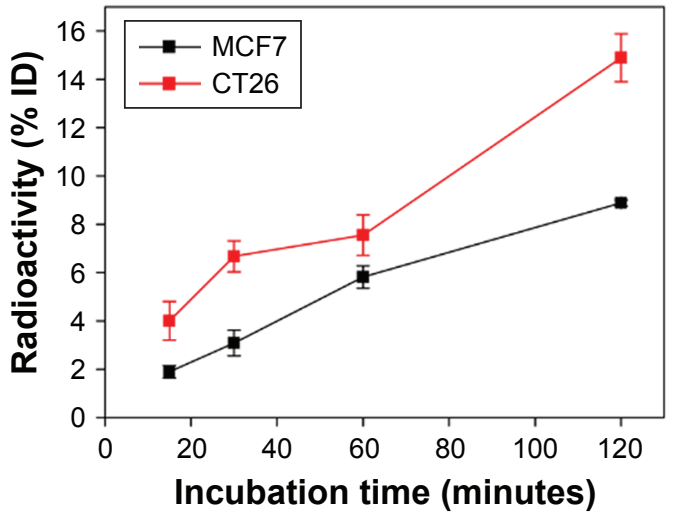

Figure 6 Radio-labeling efficiency and cell uptake studies of ${ }^{18} \mathrm{~F}-\mathrm{nGO}-\mathrm{PEG}$.

Notes: (A) Radio-thin-layer chromatography of ${ }^{18} \mathrm{~F}$-nGO-PEG and free ${ }^{18} \mathrm{~F}$. (B) Measurement of ${ }^{18} \mathrm{~F}-\mathrm{nGO}-\mathrm{PEG}$ activity in MCF7 and CT26 cells was determined by cellular uptake of ${ }^{18} \mathrm{~F}-\mathrm{nGO}-\mathrm{PEG}$ according to incubation time. All data expressed as means of triplicate experiments. Abbreviations: $n G O$, nano-graphene oxide; PEG, polyethylene glycol; ID, injected dose. 
A

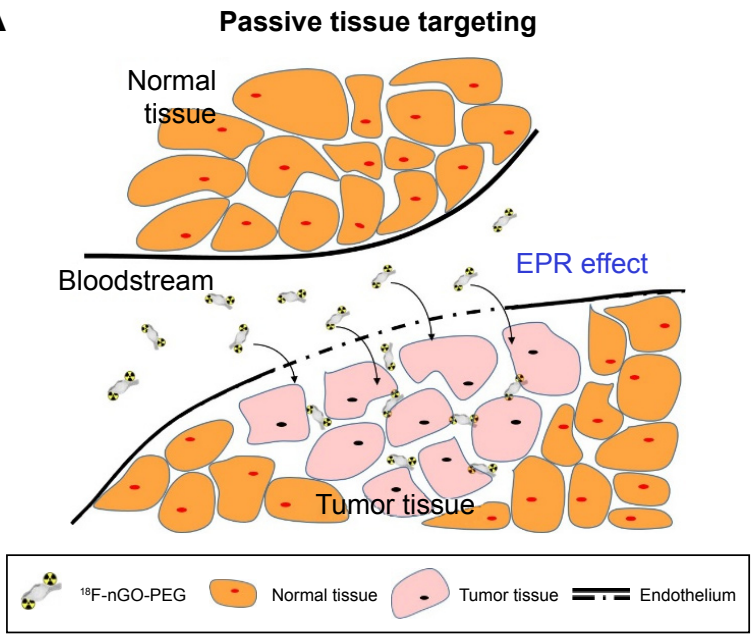

B 18 F-nGO-PEG

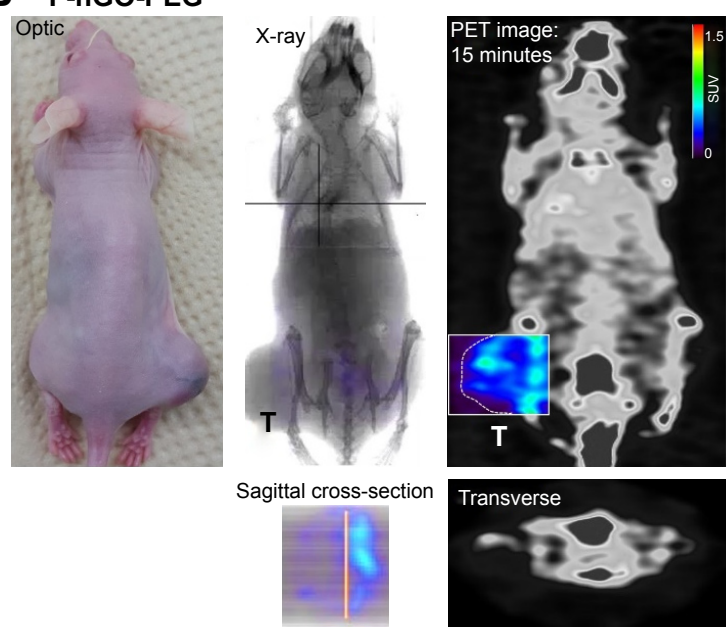

Figure 7 Illustration and bioapplications of ${ }^{18} \mathrm{~F}-\mathrm{nGO}-\mathrm{PEG}$.

Notes: (A) Representation of different mechanisms by which imaging agents can be delivered to tumors. ${ }^{18} \mathrm{~F}-\mathrm{nGO}-\mathrm{PEG}$ is shown as a representative radiotracer. Passive tissue targeting is achieved by the extravasation of nanoparticles through the increased permeability of the tumor vasculature (EPR effect). (B) Representative whole-body PET image of a CT26 tumor-bearing mice at 15 minutes after an intravenous injection. The letter "T" indicates the location of the tumor.

Abbreviations: EPR, enhanced permeability and retention; nGO, nano-graphene oxide; PEG, polyethylene glycol; PET, positron-emission tomography.

A strong PET signal was observed at the tumor site, indicating efficient formation of ${ }^{18} \mathrm{~F}-\mathrm{nGO}-\mathrm{PEG}$. The clear tumor image appeared within 15 minutes postinjection, suggesting that ${ }^{18} \mathrm{~F}-\mathrm{nGO}-\mathrm{PEG}$ accumulated rapidly in tumors through the EPR effect. Commonly, a nanomaterial (10-100 nm) can extravasate through the endothelial cell layers and interact with the cell structures of various tissues due to the EPR effect. The as-prepared ${ }^{18} \mathrm{~F}-\mathrm{nGO}-\mathrm{PEG}$ offers a large surface area, which can be loaded easily with a large amount of drugs with a variety of diagnostic and/or therapeutic agents as theranostics for disease detection and treatment. ${ }^{18} \mathrm{~F}-\mathrm{nGO}-$ PEG accumulated specifically in CT26 tumor-bearing mice via the EPR effect.

An index commonly used in clinical practice is the standardized uptake value (SUV), a normalized measure of radioactivity concentration. The SUV is now probably the most widely used method for the quantification of PET studies. The SUV represents the ${ }^{18} \mathrm{~F}-\mathrm{nGO}-\mathrm{PEG}$ uptake within a tumor. SUV is defined in the equation:

$$
\begin{aligned}
& \mathrm{SUV}= \\
& \frac{\text { Average value of tracer concentration within the target ROI }}{\text { Injection dose }(\mu \mathrm{Ci}) / \text { Body weight }(\mathrm{g})}
\end{aligned}
$$

where the radioactivity concentration is taken from a region of interest (tissue) on the PET image. SUVs obtained by tracers were calculated (Table 1). SUVs were obtained from tumor regions in PET images of free ${ }^{18} \mathrm{~F},{ }^{18} \mathrm{~F}-\mathrm{nGO}$, and ${ }^{18} \mathrm{~F}$-nGO-PEG. ${ }^{18} \mathrm{~F}$-nGO-PEG SUVs were significantly higher than Free ${ }^{18} \mathrm{~F}$ or ${ }^{18} \mathrm{~F}-\mathrm{nGO}$. Also, SUVs of free ${ }^{18} \mathrm{~F}$ and ${ }^{18} \mathrm{~F}$-nGO decreased over time. However, our material $\left({ }^{18} \mathrm{~F}\right.$ nGO-PEG) showed no change in tumor uptake over time. Validation of SUVs obtained from ${ }^{18} \mathrm{~F}-\mathrm{nGO}-\mathrm{PEG}$ against those from free ${ }^{18} \mathrm{~F}$ and ${ }^{18} \mathrm{~F}-\mathrm{nGO}$ provide evidence that PET imaging of ${ }^{18} \mathrm{~F}$-nGO-PEG may also be helpful. This technology is expected to be useful for many scientists in improving their understanding or detection of early stages of tumors in the biomedical field.

\section{Conclusion}

This paper described a green approach for the fabrication of nGO-PEG by mixing nGO with PEG under basic $\mathrm{pH}$ conditions. The as-obtained PEGylated $\mathrm{nGO}$ radiotracer has excellent stability in PBS and cell medium because of its biocompatibility and outstanding membrane-forming capability of PEG. The cytotoxicity assay showed that nGO-PEG has excellent biocompatibility in animal cell lines. Based on the results, ${ }^{18} \mathrm{~F}-\mathrm{nGO}-\mathrm{PEG}$ is believed to be a potential radiophar-

Table I SUV measurements in the tumors: statistical analysis of differences and correlations among free ${ }^{18} \mathrm{~F},{ }^{18} \mathrm{~F}-\mathrm{nGO}$, and ${ }^{18} \mathrm{~F}$ nGO-PEG

\begin{tabular}{llll}
\hline Time (minutes) & Free ${ }^{18} \mathbf{F}$ & ${ }^{18} \mathbf{F}-n G O$ & ${ }^{18}$ F-PEG-nGO \\
\hline 15 & 0 & 0.1227 & 0.2125 \\
30 & 0 & 0.1507 & 0.2731 \\
60 & 0 & 0.0912 & 0.2109
\end{tabular}

Abbreviations: nGO, nano-graphene oxide; PEG, polyethylene glycol; SUV, standardized uptake value. 
maceutical for detecting CT26 tumors and imaging tumors. The radiolabeled ${ }^{18} \mathrm{~F}$ is easily attached to the nGO-PEG surface, because it has a unique chemical structure and stability. The biocompatibility of nGO-PEG shows no obvious toxic effects on the cell. Based on this concept, radiolabeled ${ }^{18} \mathrm{~F}$ nGO-PEG is a promising radiotracer for use in the effective detection of CT26 tumors and tracking of tumor cells through imaging. The as-assembled ${ }^{18} \mathrm{~F}-\mathrm{nGO}-\mathrm{PEG}$ suggests the possibility of accurate quantification, operational simplicity, lower-cost, rapid screening that is currently unachievable by PET imaging. For this approach, radiolabeling nGO-PEG could be a potential tool for the early detection of tumors and could be used extensively in drug-delivery, bioimaging, and biomedical applications.

\section{Acknowledgments}

This work was supported by the Radiation Fusion Technology Program (2015M2A2A6A02045262(3)) from the Nuclear Research R\&D Program through the National Research Foundation of Korea (NRF), funded by the Ministry of Science and ICT, Republic of Korea. It was also partially supported from the Nuclear Research R\&D Program (2015M2B2A4028536 and 2016M2B2A4912204) through the National Research Foundation of Korea (NRF), funded by the Ministry of Science and ICT, Republic of Korea. Special thanks is given to Dr Min Goo Hur, team leader of Cyclotron from Radiation Instrumentation Research Division and Dr Jin-Woo Lee from Safety Management Team, KAERI for their kind support in facility use.

\section{Disclosure}

The authors report no conflicts of interest in this work.

\section{References}

1. Jemal A, Bray F, Center MM, Ferlay J, Ward E, Forman D. Global cancer statistics. CA Cancer J Clin. 2011;61(2):69-90.

2. Fass L. Imaging and cancer: a review. Mol Oncol. 2008;2(2):115-152.

3. Servant A, Jacobs I, Bussy C, et al. Gadolinium-functionalised multiwalled carbon nanotubes as a T1 contrast agent for MRI cell labelling and tracking. Carbon. 2016;97:126-133.

4. Phelps ME. Positron emission tomography provides molecular imaging of biological processes. Proc Natl Acad Sci U S A. 2000;97(16): 9226-9233.

5. Ametamey SM, Honer M, Schubiger PA. Molecular imaging with PET. Chem Rev. 2008;108(5):1501-1516.

6. Weissleder R. Molecular imaging in cancer. Science. 2006;312(5777): 1168-1171.

7. Huiban M, Tredwell M, Mizuta S, et al. A broadly applicable $\left[{ }^{18} \mathrm{~F}\right]$ trifluoromethylation of aryl and heteroaryl iodides for PET imaging. Nat Chem. 2013;5(11):941-944.

8. Wang M, Gao M, Mock BH, et al. Synthesis of carbon-11 labeled fluorinated 2-arylbenzothiazoles as novel potential PET cancer imaging agents. Bioorg Med Chem. 2006;14(24):8599-8607.
9. Gambhir SS. Molecular imaging of cancer with positron emission tomography. Nat Rev Cancer. 2002;2(9):683-693.

10. Hong $\mathrm{H}$, Zhang $\mathrm{Y}$, Engle JW, et al. In vivo targeting and positron emission tomography imaging of tumor vasculature with ${ }^{66} \mathrm{Ga}$-labeled nano-graphene. Biomaterials. 2012;33(16):4147-4156.

11. Sun X, Huang X, Yan X, et al. Chelator-free ${ }^{64} \mathrm{Cu}$-integrated gold nanomaterials for positron emission tomography imaging guided photothermal cancer therapy. ACS Nano. 2014;8(8):8438-8446.

12. Choi JS, Park JC, Nah H, et al. A hybrid nanoparticle probe for dualmodality positron emission tomography and magnetic resonance imaging. Angew Chem Int Ed Engl. 2008;47(33):6259-6262.

13. Peer D, Karp JM, Hong S, Farokhzad OC, Margalit R, Langer R. Nanocarriers as an emerging platform for cancer therapy. Nat Nanotechnol. 2007;2(12):751-760.

14. Pham TN, Lengkeek NA, Greguric I, et al. Tunable and noncytotoxic PET/sPECT-MRI multimodality imaging probes using colloidally stable ligand-free superparamagnetic iron oxide nanoparticles. Int $J$ Nanomedicine. 2017;12:899-909.

15. Matsumura Y, Maeda H. A new concept for macromolecular therapeutics in cancer chemotherapy: mechanism of tumoritropic accumulation of proteins and the antitumor agent Smancs. Cancer Res. 1986;46(12 Pt 1): 6387-6392.

16. Yuan F, Dellian M, Fukumura D, et al. Vascular permeability in a human tumor xenograft: molecular size dependence and cutoff size. Cancer Res. 1995;55(17):3752-3756.

17. Locatelli E, Gil L, Israel LL, et al. Biocompatible nanocomposite for PET/MRI hybrid imaging. Int J Nanomedicine. 2012;7:6021-6033.

18. Torchilin VP. Recent advances with liposomes as pharmaceutical carriers. Nat Rev Drug Discov. 2005;4(2):145-160.

19. Yang K, Li Y, Tan X, Peng R, Liu Z. Behavior and toxicity of graphene and its functionalized derivatives in biological systems. Small. 2013;9(9-10):1492-1503.

20. Shen H, Zhang L, Liu M, Zhang Z. Biomedical applications of graphene. Theranostics. 2012;2(3):283-294.

21. Zhang Y, Nayak TR, Hong H, Cai W. Graphene: a versatile nanoplatform for biomedical applications. Nanoscale. 2012;4(13):3833-3842.

22. Feng L, Liu Z. Graphene in biomedicine: opportunities and challenges. Nanomedicine (Lond). 2011;6(2):317-324.

23. Yang K, Feng L, Shi X, Liu Z. Nano-graphene in biomedicine: theranostic applications. Chem Soc Rev. 2013;42(2):530-547.

24. Feng L, Wu L, Qu X. New horizons for diagnostics and therapeutic applications of graphene and graphene oxide. Adv Mater. 2013;25(2): 168-186.

25. Bitounis D, Ali-Boucetta H, Hong BH, Min DH, Kostarelos K. Prospects and challenges of graphene in biomedical applications. Adv Mater. 2013;25(16):2258-2268.

26. Rastogi RP, Singh SP, Häder DP, Sinha RP. Detection of reactive oxygen species (ROS) by the oxidant-sensing probe $2^{\prime}, 7^{\prime}$-dichlorodihydrofluorescein diacetate in the cyanobacterium Anabaena variabilis PCC 7937. Biophys Res Commun. 2010;397(3):603-607.

27. Kim KI, Jang SJ, Park JH, et al. Detection of increased ${ }^{64} \mathrm{Cu}$ uptake by human copper transporter 1 gene overexpression using PET with ${ }^{64} \mathrm{CuCl}_{2}$ in human breast cancer xenograft model. $J$ Nucl Med. 2014;55(10):1692-1698.

28. Hess E, Takács S, Scholten B, Tárkányi F, Coenen HH, Qaim SM. Excitation function of the ${ }^{18} \mathrm{O}(\mathrm{p}, \mathrm{n})^{18} \mathrm{~F}$ nuclear reaction from threshold up to $30 \mathrm{MeV}$. Radiochim Acta. 2001;89(6):357-362.

29. Zhang S, Yang K, Feng L, Liu Z. In vitro and in vivo behaviors of dextran functionalized graphene. Carbon. 2011;49(12):4040-4049.

30. Yang K, Feng L, Hong H, Cai W, Liu Z. Preparation and functionalization of graphene nanocomposites for biomedical applications. Nat Protoc. 2013;8(12):2392-2403.

31. Kam NWS, Liu Z, Dai H. Functionalization of carbon nanotubes via cleavable disulfide bonds for efficient intracellular delivery of siRNA and potent gene silencing. J Am Chem Soc. 2005;127(36):12492-12493. 


\section{Supplementary materials}

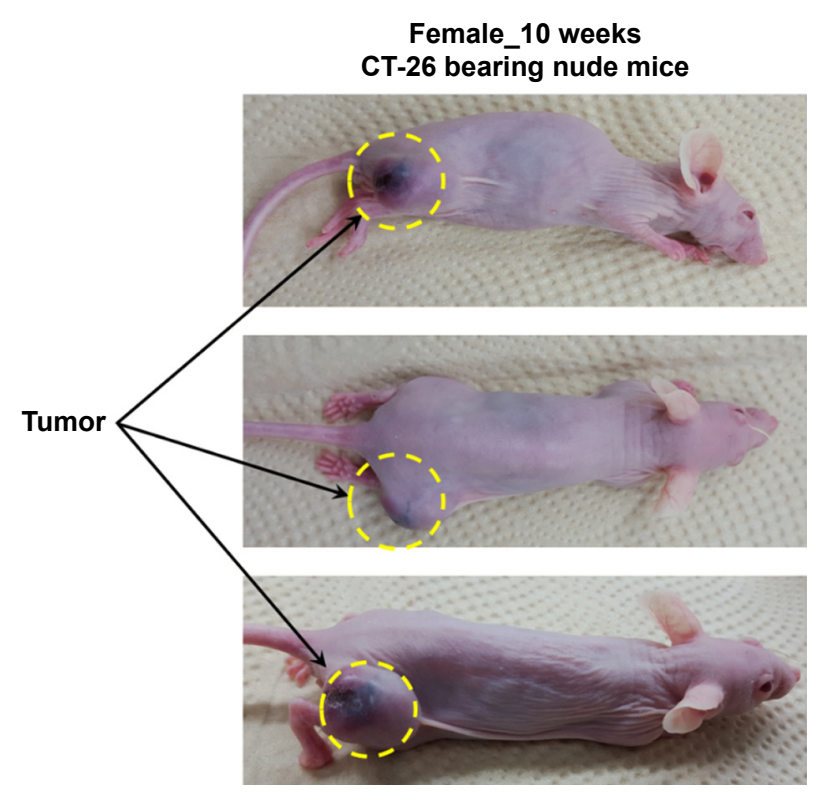

Figure SI CT26-bearing nude mice.

Note: Tumors were grown in the right thighs of the nude mice $(20-25 \mathrm{~g})$ by injecting tumor cells.
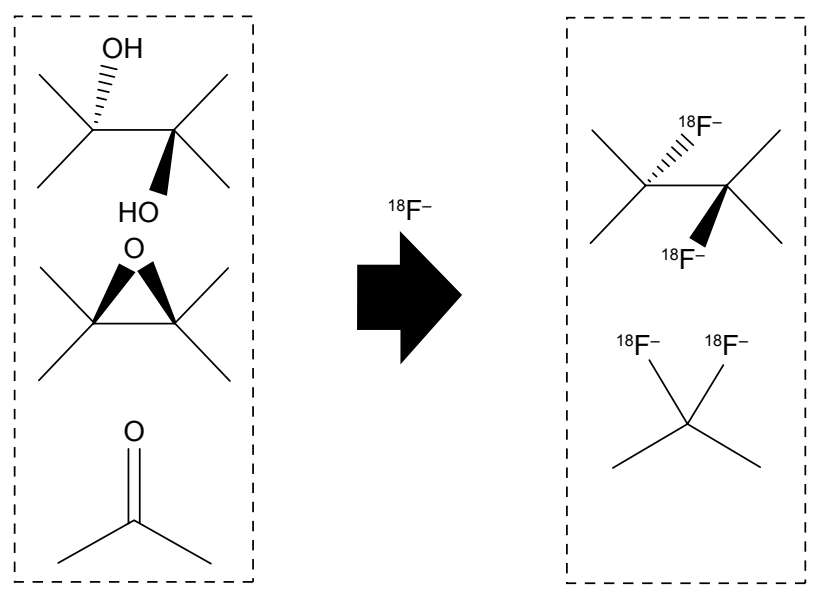

Figure S2 Conjugation chemistry between $\mathrm{nGO}$ and ${ }^{18} \mathrm{~F}^{-}$.

Notes: The ${ }^{18} \mathrm{~F}^{-}$is connected to the $\mathrm{nGO}$. The hydrogen atoms bonding on the organic macromolecules $(\mathrm{C}-\mathrm{H}$ and $\mathrm{O}-\mathrm{H})$ are easily substituted by fluorine atoms. Abbreviation: $\mathrm{nGO}$, nano-graphene oxide. 


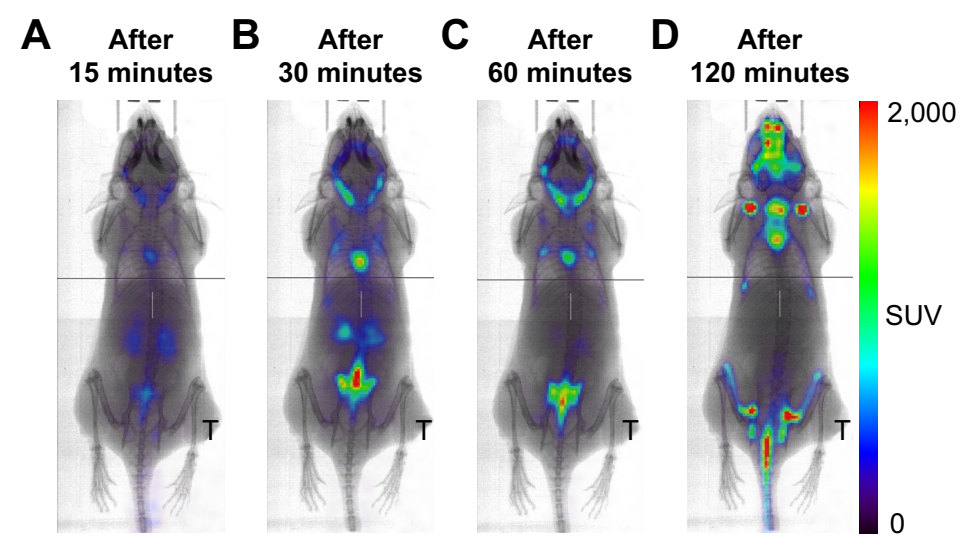

Figure S3 ${ }^{18} \mathrm{~F}$ was injected intravenously into the female mice and images monitored at different times.

Note: PET images of tumor-bearing mice at (A) 15, (B) 30, (C) 60, and (D) 120 min after intravenous injection. No signal was observed in tumor cells under in vivo conditions. Abbreviation: SUV, standardized uptake value.

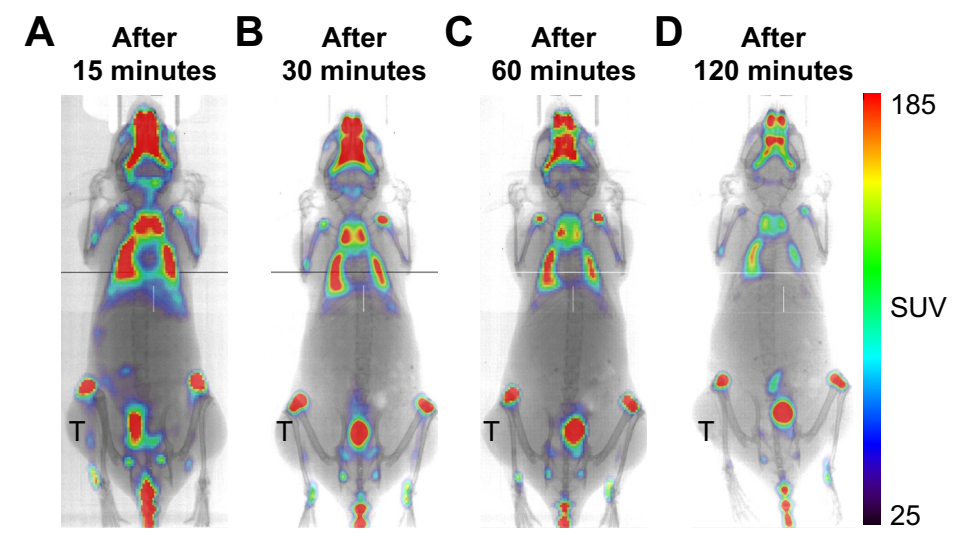

Figure S4 ${ }^{18} \mathrm{~F}-\mathrm{nGO}$ was injected intravenously into the female mice and images monitored at different times.

Note: PET images of tumor-bearing mice at (A) 15, (B) 30, (C) 60, and (D) 120 min after intravenous injection. No signal was observed in tumor cells under in vivo conditions. Abbreviation: SUV, standardized uptake value.

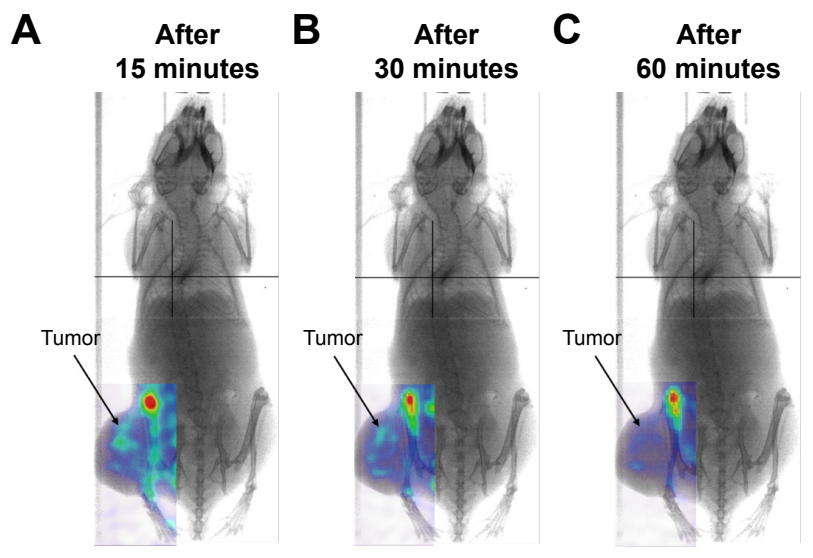

Figure S5 ${ }^{18} \mathrm{~F}$-nGO-PEG was injected intravenously into the female mice and images monitored at different times (I5, 30, and 60 minutes).

Notes: An intact ${ }^{18} \mathrm{~F}-\mathrm{nGO}-\mathrm{PEG}$ tumor and bioimaging of tumor cell were monitored. PET images of tumor-bearing mice at (A) I5, (B) 30, and (C) 60 after intravenous injection. CT26 was taken up rapidly into the early endosomes and lysosomes of CT26 tumor cells within 15 minutes.

Abbreviations: $\mathrm{nGO}$, nano-graphene oxide; PEG, polyethylene glycol. 


\section{Publish your work in this journal}

The International Journal of Nanomedicine is an international, peerreviewed journal focusing on the application of nanotechnology in diagnostics, therapeutics, and drug delivery systems throughout the biomedical field. This journal is indexed on PubMed Central, MedLine, CAS, SciSearch $\AA$, Current Contents $\AA /$ Clinical Medicine,

Journal Citation Reports/Science Edition, EMBase, Scopus and the Elsevier Bibliographic databases. The manuscript management system is completely online and includes a very quick and fair peer-review system, which is all easy to use. Visit http://www.dovepress.com/ testimonials.php to read real quotes from published authors.

Submit your manuscript here: http://www.dovepress.com/international-journal-of-nanomedicine-journal 